Pure and Applied Mathematics Quarterly

Volume 3, Number 1

(Special Issue: In honor of

Robert MacPherson, Part 3 of 3)

$1-24,2007$

\title{
On the Equivalence of Geometric and Analytic K-Homology
}

\author{
Paul Baum, Nigel Higson, and Thomas Schick
}

\begin{abstract}
We give a proof that the geometric K-homology theory for finite CWcomplexes defined by Baum and Douglas is isomorphic to Kasparov's K-homology. The proof is a simplification of more elaborate arguments which deal with the geometric formulation of equivariant K-homology theory.
\end{abstract}

\section{INTRODUCTION}

K-homology theory, the homology theory which is dual to Atiyah-Hirzebruch Ktheory, may be defined abstractly using the Bott spectrum and standard contructions in homotopy theory. Atiyah [Ati70] pointed out the relevance to index theory of a concrete definition of K-homology. Following his suggestions, detailed analytic definitions of Khomology were provided by Brown, Douglas and Fillmore [BDF77] and by Kasparov [Kas75], and these works are now foundational papers in operator K-theory. At about the same time, Baum and Douglas [BD82] introduced a geometric definition of K-homology (using manifolds, bordisms, and so on) in connection with work on the Riemann-Roch problem [BFM75, BFM79]. Baum and Douglas defined a very simple and natural map from their geometric theory to analytic K-homology, and this map turns out to be an isomorphism. The combined efforts of various mathematicians in the early 1980's produced a proof of this, but a detailed account of the matter was never published. This is despite the fact that over the years the isomorphism has grown in importance, thanks to its connection with the Baum-Connes conjecture [BCH94]. The purpose of this note is to present, after a twenty five year gap, a detailed proof of the isomorphism from geometric K-homology to analytic K-homology. (See [Jak98, Jak00] for a related approach to the problem of defining homology theories dual to multiplicative cohomology theories like K-theory.) The proof is a spin-off from our work on equivariant K-homology theory, which will be reported upon in a future paper, where we shall prove that for a discrete, countable group G, geometric equivariant K-homology is isomorphic to analytic equivariant K-homology on the category of proper, finite G-CW-complexes.

Received December 18, 2005. 
With admiration and affection we dedicate this paper to Robert MacPherson. A conversation between the first-named author and Bob MacPherson at IHES in 1978 was crucial to the eventual formulation of geometric K-homology.

\section{ReView of AnAlytic K-Homology}

In this section we shall review Kasparov's definition of analytic K-homology, and list those facts about it that we shall need in the sequel. For further details the reader is referred to the monograph [HR00] on the subject.

Throughout this section we shall be working with locally compact, second countable topological spaces. If $Z$ is such a space then we shall denote by $C_{0}(Z)$ the (separable) $C^{*}$-algebra of continuous, complex-valued functions on $Z$ which vanish at infinity.

If $X$ and $Y$ are operators on a Hilbert space, then the notation $X \sim Y$ will signify the equality of $X$ and $Y$ modulo the compact operators.

Definition 2.1. Let $A$ be a separable $C^{*}$-algebra. An (ungraded) Fredholm module over $A$ is given by the following data:

(a) a separable Hilbert space $\mathrm{H}$,

(b) a representation $\rho: A \rightarrow \mathcal{B}(H)$ of $A$ as bounded operators on $H$, and

(c) an operator $F$ on $\mathrm{H}$ such that for all $a \in A$,

$$
\left(F^{2}-1\right) \rho(a) \sim 0, \quad\left(F-F^{*}\right) \rho(a) \sim 0, \quad F \rho(a) \sim \rho(a) F .
$$

The representation $\rho$ is not required to be non-degenerate in any way. In fact $\rho$, and even the Hilbert space $\mathrm{H}$, are allowed to be zero.

Roughly speaking, Kasparov's K-homology groups are assembled from homotopy classes of Fredholm modules over $A=C_{0}(Z)$. However it is necessary to equip these Fredholm modules with a modest amount of extra structure.

Definition 2.2. Let $p \in\{0,1,2, \ldots\}$ and let $A$ be a separable $C^{*}$-algebra. A p-graded ${ }^{1}$ Fredholm module is a Fredholm module $(H, \rho, F)$, as above, with the following additional structure:

(a) The Hilbert space $\mathrm{H}$ is equipped with a $\mathbb{Z} / 2$-grading $\mathrm{H}=\mathrm{H}^{+} \oplus \mathrm{H}^{-}$in such a way that for each $a \in A$, the operator $\rho(a)$ is even-graded, while the operator $F$ is odd-graded.

(b) There are odd-graded operators $\varepsilon_{1}, \ldots, \varepsilon_{p}$ on $\mathrm{H}$ such that

$$
\varepsilon_{j}=-\varepsilon_{j}^{*}, \quad \varepsilon_{j}^{2}=-1, \quad \varepsilon_{i} \varepsilon_{j}+\varepsilon_{j} \varepsilon_{i}=0 \quad(i \neq j),
$$

and such that $F$ and each $\rho(a)$ commute with each $\varepsilon_{j}$.

\footnotetext{
${ }^{1}$ The term ' $p$-multigraded' is used in [HR00].
} 
Of course, if $p=0$ then part (b) of the definition does not apply.

Definition 2.3. Let $(H, \rho, F)$ and $\left(H^{\prime}, \rho^{\prime}, F^{\prime}\right)$ be $p$-graded Fredholm modules over $A$. A unitary equivalence between them is a grading-degree zero unitary isomorphism $\mathrm{U}: \mathrm{H} \rightarrow$ $\mathrm{H}^{\prime}$ which intertwines the representations $\rho$ and $\rho^{\prime}$, the operators $F$ and $F^{\prime}$, and the grading operators $\varepsilon_{j}$ and $\varepsilon_{j}^{\prime}$.

Definition 2.4. Suppose that $\left(H, \rho, F_{t}\right)$ is a family of $p$-graded Fredholm modules parameterized by $t \in[0,1]$, in which the representation $\rho$, the Hilbert space $H$ and its grading structures remain constant but the operator $F_{t}$ varies with $t$. If the function $t \mapsto F_{t}$ is norm continuous, then we say that the family defines an operator homotopy between the $p$-graded Fredholm modules $\left(\rho, H, F_{0}\right)$ and $\left(\rho, H, F_{1}\right)$, and that the two Fredholm modules are operator homotopic.

There is a natural notion of direct sum for Fredholm modules: one takes the direct sum of the Hilbert spaces, of the representations, and of the operators F. The zero module has zero Hilbert space, zero representation, and zero operator.

Now we can give Kasparov's definition of K-homology.

Definition 2.5. Let $p \in\{0,1,2, \ldots\}$ and let $A$ be a separable $C^{*}$-algebra. The Kasparov $\mathrm{K}$-homology group $\mathrm{K}^{-\mathrm{p}}(\mathrm{A})$ is the abelian group with one generator $[\mathrm{x}]$ for each unitary equivalence class of $p$-graded Fredholm modules over $A$ and with the following relations:

(a) if $x_{0}$ and $x_{1}$ are operator homotopic $p$-graded Fredholm modules then $\left[x_{0}\right]=\left[x_{1}\right]$ in $\mathrm{K}^{-\mathrm{p}}(\mathrm{A})$, and

(b) if $x_{0}$ and $x_{1}$ are any two $p$-graded Fredholm modules then $\left[x_{0} \oplus x_{1}\right]=\left[x_{0}\right]+\left[x_{1}\right]$ in $\mathrm{K}^{-\mathrm{p}}(\mathrm{A})$.

Definition 2.6. A p-graded Fredholm module is said to be degenerate if the equivalences modulo compact operators listed in item (c) of Definition 2.1 are actually equalities.

It is easy to see that a degenerate $p$-graded Fredholm module determines the zero element of $K^{-p}(A)$.

Lemma 2.7. Let $(\mathrm{H}, \rho, \mathrm{F})$ be a $\mathrm{p}$-graded Fredholm module. Assume that there exists a self-adjoint, odd-graded involution $\mathrm{E}: \mathrm{H} \rightarrow \mathrm{H}$ which commutes with the action of $\mathrm{A}$ and with the multigrading operators $\varepsilon_{\mathfrak{j}}$, and which anticommutes with $\mathrm{F}$. Then the Fredholm module $(H, \rho, F)$ represents the zero element of $\mathrm{K}^{-\mathrm{p}}(\mathrm{A})$.

Proof. The path $\mathrm{F}_{\mathrm{t}}=\cos (\mathrm{t}) \mathrm{F}+\sin (\mathrm{t}) \mathrm{E}$ gives an operator homotopy from $\mathrm{F}$ to the degenerate operator $\mathrm{E}$.

It follows from the lemma that the additive inverse of the K-homology class represented by $(H, \rho, F)$ is the class of $\left(H^{o p p}, \rho,-F\right)$, where $H^{\text {opp }}$ denotes $H$ with the grading 
reversed. This is because the involution $\left(\begin{array}{ll}0 & 1 \\ 1 & 0\end{array}\right)$ on $\mathrm{H} \oplus \mathrm{H}^{\mathrm{opp}}$ satisfies the hypotheses of the lemma, applied to the Fredholm module $\left(H \oplus H^{\text {opp }}, \rho \oplus \rho, F \oplus-F\right)$. It follows that every class in $K^{-p}(A)$ is represented by a single Fredholm module, and that two modules represent the same class if and only if, up to isomorphism, they become operator homotopic after adding degenerate modules.

If $(H, \rho, F)$ is a $p$-graded Fredholm module $A$, then we may construct from it a $(p+2)$ graded Fredholm module $\left(H^{\prime}, \rho^{\prime}, F^{\prime}\right)$ over $A$ by means of the formulas

$$
\mathrm{H}^{\prime}=\mathrm{H} \oplus \mathrm{H}^{\mathrm{opp}}, \quad \rho^{\prime}=\rho \oplus \rho, \quad \mathrm{F}^{\prime}=\mathrm{F} \oplus \mathrm{F},
$$

along with the grading operators

$$
\varepsilon_{j}=\varepsilon_{j} \oplus \varepsilon_{j} \quad(j=1, \ldots p), \quad \varepsilon_{p+1}=\left(\begin{array}{cc}
0 & I \\
-I & 0
\end{array}\right) \quad \text { and } \quad \varepsilon_{p+2}=\left(\begin{array}{cc}
0 & i I \\
i I & 0
\end{array}\right) .
$$

Definition 2.8. The formal periodicity map

$$
\mathrm{K}^{-\mathrm{p}}(\mathrm{A}) \rightarrow \mathrm{K}^{-(\mathrm{p}+2)}(\mathrm{A})
$$

is the homomorphism of Kasparov groups induced from this construction.

The periodicity map can be reversed by compressing a $(p+2)$ graded Fredholm module to the +1 eigenspace of the involution $-i \varepsilon_{p+1} \varepsilon_{p+2}$. We obtain an isomorphism

$$
\mathrm{K}^{-\mathrm{p}}(\mathrm{A}) \cong \mathrm{K}^{-(\mathrm{p}+2)}(\mathrm{A}) \text {. }
$$

As a result there are really only two genuinely distinct $\mathrm{K}$-homology groups, $\mathrm{K}^{\mathrm{ev}}$ and $\mathrm{K}^{\text {odd }}$, as follows:

Definition 2.9. Let us denote by $K^{\mathrm{ev}}(A)$ and $K^{\text {odd }}(A)$ the groups $K^{0}(A)$ and $K^{-1}(A)$ respectively, or more canonically, the direct limits

$$
K^{\mathrm{ev}}(A)=\underset{\mathrm{k}}{\lim } K^{-2 \mathrm{k}}(A) \quad \text { and } \quad K^{\text {odd }}(A)=\underset{\mathrm{k}}{\lim } K^{-(1+2 k)}(A)
$$

under the above periodicity maps.

Definition 2.10. If $Z$ is a second countable, ${ }^{2}$ locally compact space, and if $A=C_{0}(Z)$, then we shall write $K_{p}(Z)$ in place of $K^{-p}(A)$. These are the Kasparov K-homology groups of the space $Z$. If $(X, Y)$ is a second countable, locally compact pair, and if $Z$ is the difference $X \backslash Y$, then we define relative $K$-homology groups by

$$
K_{p}(X, Y)=K_{-p}(Z)
$$

We shall define periodic groups $K_{\text {ev }}$ odd $(X, Y)$ similarly.

Kasparov's main theorem concerning these objects is then as follows:

\footnotetext{
${ }^{2}$ This assumption is required at several points in Kasparov's theory, which is designed for separable $\mathrm{C}^{*}$-algebras.
} 
Theorem 2.11. There are natural transformations

$$
\partial: \mathrm{K}_{\mathrm{p}}(\mathrm{X}, \mathrm{Y}) \rightarrow \mathrm{K}_{\mathrm{p}-1}(\mathrm{Y})
$$

(connecting homomorphisms) which are compatible with the formal periodicity isomorphisms and which give Kasparov K-homology the structure of a $\mathbb{Z} / 2$-graded homology theory on the category of compact metrizable pairs $(\mathrm{X}, \mathrm{Y})$. On the subcategory of finite $\mathrm{CW}$-complexes Kasparov K-homology is isomorphic to topological K-homology - the homology theory associated to the Bott spectrum.

\section{DIRAC-TYPE OPERATORS}

We continue to follow the monograph [HR00].

Definition 3.1. Let $M$ be a smooth, second countable finite dimensional manifold (possibly with non-empty boundary) and let $\mathrm{V}$ be a smooth, Euclidean vector bundle over $M$. A p-graded Dirac structure on $V$ is a smooth, $\mathbb{Z} / 2$-graded, Hermitian vector bundle $S$ over $M$ together with the following data:

(a) An $\mathbb{R}$-linear morphism of vector bundles

$$
\mathrm{V} \rightarrow \operatorname{End}(\mathrm{S})
$$

which associates to each vector $v \in V_{x}$ a skew-adjoint, odd-graded endomorphism $u \mapsto v \cdot u$ of $S_{x}$ in such a way that

$$
v \cdot v \cdot u=-\|v\|^{2} u
$$

(b) A family of skew-adjoint, odd-graded endomorphisms $\varepsilon_{1}, \ldots, \varepsilon_{p}$ of $S$ such that

$$
\varepsilon_{j}=-\varepsilon_{j}^{*}, \quad \varepsilon_{j}^{2}=-1, \quad \varepsilon_{i} \varepsilon_{j}+\varepsilon_{j} \varepsilon_{i}=0 \quad(i \neq j),
$$

and such that each $\varepsilon_{j}$ commutes with each operator $u \mapsto v \cdot u$.

Usually $M$ will be a Riemannian manifold and we will take $V=T M$. In this case we shall call $S$ a $p$-graded Dirac bundle on $\mathrm{M}$.

Definition 3.2. Let $M$ be a Riemannian manifold which is equipped with a p-graded Dirac structure, with Dirac bundle $S$. We shall call an odd-graded, symmetric, order one linear partial differential operator $\mathrm{D}$ acting on the sections of $S$ a Dirac operator if it commutes with the operators $\varepsilon_{j}$, and if

$$
[D, f] u=\operatorname{grad} f \cdot u,
$$

for every smooth function $f$ on $M$ and every section $u$ of $S$.

Every Dirac bundle on a Riemannian manifold admits a Dirac operator, and the difference of two Dirac operators on a single Dirac bundle $S$ is an endomorphism of $S$.

A $p$-graded Dirac operator $D$ on a Riemannian manifold $M$ without boundary defines in a natural way a class $[D] \in K_{p}(M)$. The general construction is a little involved, and 
we refer the reader to [HR00] for details, but when $M$ is closed there is a very simple description of $[D]$ :

Theorem 3.3. Let $\mathrm{M}$ be a closed (i.e. compact without boundary) Riemannian manifold and let $\mathrm{D}$ be a Dirac operator on a $\mathrm{p}$-graded Dirac bundle S. Let $\mathrm{H}=\mathrm{L}^{2}(\mathrm{M}, \mathrm{S})$ be the Hilbert space of square-integrable sections of $\mathrm{S}$, and let $\rho$ be the representation of $\mathrm{C}(\mathrm{M})$ on $\mathrm{H}$ by pointwise multiplication operators. Let

$$
\mathrm{F}=\mathrm{D}\left(\mathrm{I}+\mathrm{D}^{2}\right)^{-\frac{1}{2}} .
$$

The triple $(\rho, \mathrm{H}, \mathrm{F})$ is a $\mathrm{p}$-graded Fredholm module for $\mathrm{A}=\mathrm{C}(\mathrm{M})$.

To describe further properties of the classes $[D]$ we need to introduce the following boundary operation on Dirac bundles:

Definition 3.4. Let $S$ be a $p$-graded Dirac bundle on a Riemannian manifold $\bar{M}$ with boundary $\partial M$. If $e_{1}$ denotes the outward pointing unit normal vector field on the boundary manifold $\partial M$ then the formula

$$
X: u \mapsto(-1)^{\partial u} e_{1} \cdot \varepsilon_{1} u
$$

defines an automorphism of the restriction of $S$ to $\partial M$ which is even, self-adjoint, and satisfies $X^{2}=1$. The operator $X$ commutes with multiplication $u \mapsto Y \cdot u$ by tangent vectors $Y$ orthogonal to $e_{1}$, and also with the multigrading operators $\varepsilon_{2}, \ldots, \varepsilon_{p}$. The +1 eigenbundle for $X$ is a $(p-1)$-graded Dirac bundle ${ }^{3}$ on $\partial M$, which we shall call the boundary of the Dirac bundle $S$.

The following theorem summarizes facts proved in Chapters 10 and 11 of [HR00].

Theorem 3.5. To each Dirac operator D on a p-graded Dirac bundle over a smooth manifold without boundary there is associated a class $[\mathrm{D}] \in \mathrm{K}_{\mathrm{p}}(\mathrm{M})$ with the following properties:

(i) The class [D] depends only on the Dirac bundle, not on the choice of the operator D.

(ii) If $\mathrm{M}_{1}$ is an open subset of $\mathrm{M}_{2}$, and if $\mathrm{D}_{1}$ is a Dirac operator on $\mathrm{M}_{1}$ obtained by restricting a Dirac operator $\mathrm{D}_{2}$ on $\mathrm{M}_{2}$, then $\left[\mathrm{D}_{2}\right]$ maps to $\left[\mathrm{D}_{1}\right]$ under the homomorphism $\mathrm{K}_{\mathrm{p}}\left(\mathrm{M}_{2}\right) \rightarrow \mathrm{K}_{\mathrm{p}}\left(\mathrm{M}_{1}\right)$.

(iii) Let $\mathrm{M}$ be the interior of a Riemannian manifold $\overline{\mathrm{M}}$ with boundary $\partial \mathrm{M}$, and let $\mathrm{S}$ be a $\mathrm{p}$-graded Dirac bundle on $\overline{\mathrm{M}}$. Let $\mathrm{D}$ be a Dirac operator on $\mathrm{M}$ associated to $\mathrm{S}$ and let $\mathrm{D}_{\partial \mathrm{M}}$ be a Dirac operator on $\partial \mathrm{M}$ associated to the boundary of $\mathrm{S}$. The connecting homomorphism

$$
\partial: K_{p}(M) \rightarrow K_{p-1}(\partial M)
$$

in Kasparov K-homology takes the class $\left[\mathrm{D}_{\mathrm{M}}\right]$ to the class $\left[\mathrm{D}_{\partial \mathrm{M}}\right]$ :

$$
\partial\left[D_{M}\right]=\left[D_{\partial M}\right] \in K_{p-1}(\partial M) .
$$

\footnotetext{
${ }^{3}$ The multigrading operators are obtained from $\varepsilon_{2}, \ldots, \varepsilon_{p}$ by shifting indices downwards.
} 
We shall need one additional fact about Dirac operators which concerns the structure of operators on fiber bundles. Suppose that $M$ is a closed Riemannian manifold and that $P$ is a principal bundle over $M$ whose structure group is a compact Lie group $G$. Suppose that $\mathrm{N}$ is a closed Riemannian manifold on which $\mathrm{G}$ acts by isometries. We can then form the manifold $Z=P \times_{G} N$. Its tangent bundle $T Z$ fits into an exact sequence of vector bundles over $Z$,

$$
0 \longrightarrow \mathrm{V} \longrightarrow \mathrm{TZ} \longrightarrow \pi^{*} \mathrm{TM} \longrightarrow 0
$$

where $\pi$ denotes the projection mapping from $Z$ to $M$ and where $V$ denotes the "vertical tangent bundle" $\mathrm{V}=\mathrm{P} \times_{\mathrm{G}} \mathrm{TN}$. If we choose a splitting of the sequence then we obtain an isomorphism

$$
\mathrm{TZ} \cong \mathrm{V} \oplus \pi^{*} \mathrm{TM}
$$

which equips $Z$ with a Riemannian metric.

Now suppose that $S_{M}$ is a p-graded Dirac bundle for $M$ and that $S_{N}$ is a 0 -graded Dirac bundle for $N$. Let us also suppose that there is an action of $G$ on $S_{N}$ which is compatible with the action of $\mathrm{G}$ on $\mathrm{N}$. We can then form the bundle $S_{V}=\mathrm{P} \times{ }_{\mathrm{G}} S_{\mathrm{N}}$ over $Z$, and from it the graded tensor product $S_{Z}=S_{V} \hat{\otimes} \pi^{*} S_{M}$. Using the direct sum decomposition (3.1) this becomes a $p$-graded Dirac bundle for $Z$, with the tangent vector $v \oplus w \in V \oplus \pi^{*} \mathrm{TM}$ acting as the operator $v \hat{\otimes} 1+1 \hat{\otimes} w$ on $S_{V} \hat{\otimes} \pi^{*} S_{M}$.

We can now form the class $\left[D_{Z}\right] \in K_{p}(Z)$ associated to a Dirac operator on the Dirac bundle $S_{V} \hat{\otimes} \pi^{*} S_{M}$, and using the projection mapping $\pi: Z \rightarrow M$ we obtain a class

$$
\pi_{*}\left[\mathrm{D}_{Z}\right] \in \mathrm{K}_{\mathrm{p}}(\mathrm{M}) .
$$

The following proposition relates $\pi_{*}\left[D_{Z}\right]$ to the class $\left[D_{M}\right]$ of a Dirac operator for the Dirac bundle $S_{M}$ on $M$.

Proposition 3.6. Assume that there exists a G-equivariant Dirac operator for the Dirac bundle $\mathrm{S}_{\mathrm{N}}$ on $\mathrm{N}$ whose kernel is the one-dimensional trivial representation of $\mathrm{G}$, spanned by an even-graded section of $S_{\mathrm{N}}$. Then

$$
\pi_{*}\left[D_{Z}\right]=\left[D_{M}\right] \in K_{p}(M) .
$$

Proof. Let us consider first the special case in which the principal bundle $\mathrm{P}$ is trivial: $P=G \times M$ (in this case we might as well take $G=\{e\}$ ). Then of course $Z=N \times M$. We can take the Dirac operator $\mathrm{D}_{Z}$ to be

$$
D_{Z}=D_{N} \hat{\otimes} I+I \hat{\otimes} D_{M},
$$

where $D_{N}$ is a Dirac operator for the Dirac bundle $S$ on $N$ with one-dimensional kernel, as in the statement of the proposition. Now the Hilbert space on which $D_{Z}$ acts is the tensor product

$$
\mathrm{L}^{2}\left(\mathrm{~N} \times \mathrm{M}, \mathrm{S}_{\mathrm{N}} \hat{\otimes} \mathrm{S}_{M}\right)=\mathrm{L}^{2}\left(\mathrm{~N}, \mathrm{~S}_{\mathrm{N}}\right) \hat{\otimes} \mathrm{L}^{2}\left(\mathrm{M}, \mathrm{S}_{\mathrm{M}}\right)
$$


If we split the first factor, $\mathrm{L}^{2}\left(\mathrm{~N}, \mathrm{~S}_{\mathrm{N}}\right)$, as $\operatorname{ker}\left(\mathrm{D}_{\mathrm{N}}\right)$ plus its orthogonal complement, then we obtain a corresponding direct sum decomposition of $L^{2}\left(N \times M, S_{N} \hat{\otimes} S_{M}\right)$. The operator $F_{Z}$ formed from $D_{Z}$, as in Theorem 3.3, respects this direct sum decomposition, as does the action of $C(M)$. We therefore obtain a decomposition of the Fredholm module representing $\left[\mathrm{D}_{\mathrm{Z}}\right]$ as a direct sum of two Fredholm modules. The first acts on $\operatorname{ker}\left(D_{N}\right) \otimes L^{2}\left(M, S_{M}\right) \cong L^{2}\left(M, S_{M}\right)$ and is isomorphic to the Fredholm module representing $\left[D_{M}\right]$. The second represents the zero element of $K_{p}(M)$. This follows from Lemma 2.7, since if $T$ is the partial isometry part of $D_{N}$ in the polar decomposition, and if $\gamma$ is the grading operator on $\mathrm{L}^{2}\left(\mathrm{M}, \mathrm{S}_{M}\right)$, then the odd-graded involution

$$
\mathrm{E}=\mathrm{T} \hat{\otimes} \gamma
$$

on the Hilbert space $\operatorname{ker}(D)^{\perp} \hat{\otimes} L^{2}\left(M, S_{M}\right)$ commutes with the action of $C(M)$, and with the grading operators $\varepsilon_{j}$, and anticommutes with $F_{Z}$.

The proof of the general case is similar. To begin, the Hilbert space on which $D_{Z}$ acts is naturally isomorphic to the fixed point space

$$
\left[\mathrm{L}^{2}\left(\mathrm{~N}, \mathrm{~S}_{\mathrm{N}}\right) \hat{\otimes} \mathrm{L}^{2}\left(\mathrm{P}, \pi^{*} \mathrm{~S}_{\mathrm{M}}\right)\right]^{\mathrm{G}}
$$

Denote by $\widetilde{D}_{M}$ a G-equivariant linear partial differential operator on $P$, acting on sections of $\pi^{*} S_{M}$, which is obtained as follows. Select a finite cover of $M$ by open sets $\mathrm{U}_{j}$ over which the bundle $\mathrm{P}$ is trivial, and fix isomorphisms to $\mathrm{G} \times \mathrm{U}_{j}$ over these open sets. Use the isomorphisms to define operators $\widetilde{D}_{j}$ on $\pi^{-1}\left[U_{j}\right] \subseteq P$ which act as $D$ in the $U_{j}$ direction and act as the identity in the G-direction. Select also a smooth partition of unity $\left\{\sigma_{j}^{2}\right\}$ which is subordinate to the cover. Then define $\widetilde{D}$ by averaging the sum $\sum \sigma_{j} \widetilde{D}_{j} \sigma_{j}$ over the action of $G$. Having constructed $\widetilde{D}_{M}$, we obtain a Dirac operator for $S_{M} \hat{\otimes} S_{V}$ by the formula

$$
D_{Z}=D_{N} \otimes I+I \hat{\otimes} \widetilde{D}_{M}
$$

From here the argument used in the special case may be applied verbatim.

Remark 3.7. By using some machinery the preceding result can be conceptualized and generalized as follows. If $G$ is a compact group and $A$ is a $C^{*}$-algebra equipped with an action of $G$ (for example $A=C(N)$ ), then there is a natural notion of $G$-equivariant Fredholm module, from which we may define equivariant $\mathrm{K}$-homology groups $\mathrm{K}_{\mathrm{G}}^{-\mathrm{p}}(A)$. In the commutative case these give equivariant groups $K_{p}^{G}(N)$. Now if $P$ is a principal G-bundle over $\mathrm{M}$, as above, then by elaborating on the construction of the Kasparov product (which we shall not actually use anywhere in this paper) we obtain a pairing

$$
\mathrm{K}_{0}^{\mathrm{G}}(\mathrm{N}) \otimes \mathrm{K}_{\mathrm{p}}(\mathrm{M}) \stackrel{\mu_{\mathrm{p}}}{\longrightarrow} \mathrm{K}_{\mathrm{p}}(\mathrm{Z})
$$

One can compute that the class $\left[D_{M}\right] \otimes[D]$ is mapped to $\left[D_{Z}\right]$. Next, the map which collapses $\mathrm{N}$ to a point induces a homomorphism from $\mathrm{K}_{0}^{\mathrm{G}}(\mathrm{N})$ to the coefficient group $\mathrm{K}_{0}^{\mathrm{G}}(\mathrm{pt})$, which is the representation ring of $\mathrm{G}$. From a representation of $\mathrm{G}$ and the 
principal bundle $P$ we obtain by induction a vector bundle on the space $M$. We therefore obtain a map

$$
\varepsilon: \mathrm{K}_{0}^{\mathrm{G}}(\mathrm{N}) \longrightarrow \mathrm{K}^{0}(\mathrm{M}) .
$$

Finally, the group $K_{p}(M)$ is a module over the ring $K^{0}(M)$ by the cap product between homology and cohomology. We obtain a diagram

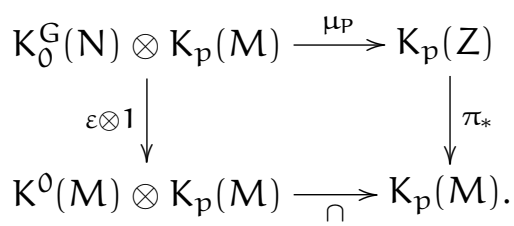

Proposition 3.6 follows from the assertion that this diagram commutes (in the special case where the collapse map sends $[D]$ to $1 \in R(G)$ ). The commutativity of the diagram is a simple exercise with the Kasparov product, but it is beyond the scope of the present article.

We conclude this section by introducing a specific Dirac operator to which we shall apply Proposition 3.6. In order to fix notation we begin with the following definition:

Definition 3.8. Let $\mathrm{V}$ be a Euclidean vector space. The complex Clifford algebra for $\mathrm{V}$ is the universal complex $*$-algebra Cliff( $(\mathrm{V})$ equipped with an $\mathbb{R}$-linear inclusion of $\mathrm{V}$, and subject to the relations $v^{2}=-\|v\|^{2} \cdot 1$ for $v \in \mathrm{V}$. If $\left\{e_{1}, \ldots, e_{\mathrm{n}}\right\}$ is an orthonormal basis for $V$, then the algebra $C$ liff $(V)$ is linearly spanned by the $2^{n}$ monomials $e_{j_{1}} \cdots e_{j_{k}}$, where $j_{1}<\cdots<j_{k}$ and $0 \leq k \leq n$. We introduce an inner product on Cliff(V) by deeming these monomials to be orthonormal.

The algebra $\operatorname{Cliff}(\mathrm{V})$ is $\mathbb{Z} / 2$-graded: the monomial $e_{j_{1}} \cdots e_{j_{k}}$ is even or odd-graded, according as $\mathrm{k}$ is even or odd.

Definition 3.9. Let $\mathrm{N}$ be an even-dimensional, Riemannian manifold and let $\mathrm{Cliff}(\mathrm{TN})$ be the complex vector bundle on $\mathrm{N}$ whose fibers are the complexified Clifford algebras of the fibers of the tangent bundle of $\mathrm{N}$. The bundle Cliff(TN) has a natural 0-graded Dirac bundle structure (tangent vectors act by Clifford multiplication on the left).

If $\mathrm{N}$ is oriented, and if $\left\{e_{1}, \ldots, e_{n}\right\}$ is a local, oriented, orthonormal frame, then the operator of right-multiplicaton by the product

$$
\sigma=i^{\frac{n}{2}} e_{1} \cdots e_{n}
$$

is an even-graded, self-adjoint involution of the bundle $\operatorname{Cliff}(\mathrm{TN})$ which commutes with the Dirac bundle structure.

Definition 3.10. Denote by $\operatorname{Cliff}_{\frac{1}{2}}(\mathrm{TN})$ the +1 -eigenbundle of the involution $\sigma$. This is a 0-graded Dirac bundle in its own right. 
We wish to compute the index of a Dirac operator associated to this Dirac bundle, at least in the case of a sphere $N=S^{n}$. To do so, we use the standard isomorphism between $\operatorname{Cliff}(T N)$ and the complexified exterior algebra bundle $\Lambda_{\mathbb{C}}^{*} T^{*} N \cong \Lambda_{\mathbb{C}}^{*} T N$, which associates to the Clifford monomial $e_{j_{1}} \cdots e_{j_{k}}$ the differential form $e_{j_{1}} \wedge \cdots \wedge e_{j_{k}}$. Under this correspondence, the operator $\mathrm{D}=\mathrm{d}+\mathrm{d}^{*}$ on forms becomes a Dirac operator for the Dirac bundle Cliff(TN). So the kernel of D is the space of harmonic forms on $\mathrm{N}$. Using the fact that the involution $\sigma$ exchanges the 0 and $\mathrm{n}$-forms on $\mathrm{N}$ we obtain the following result.

Proposition 3.11. Let $\mathrm{N}$ be an even-dimensional, round sphere (oriented as the boundary of the ball). There is a Dirac operator for $\mathrm{Cliff}_{\frac{1}{2}}(\mathrm{TN})$ which is equivariant for the natural action of the special orthogonal group, and whose kernel is the one-dimensional trivial representation, and is generated by an even-graded section of $\mathrm{Cliff}_{\frac{1}{2}}(\mathrm{TN})$.

Remark 3.12. For general oriented Riemannian manifolds $N$, the index of the Dirac operator for Cliff $\frac{1}{2}(\mathrm{TN})$ is the average of the Euler characteristic and the signature. Indeed the direct sum of Cliff $\frac{1}{2}(\mathrm{TN})$ with the opposite of the bundle complementary to $\operatorname{Cliff}_{\frac{1}{2}}(\mathrm{TN})$ in $\operatorname{Cliff}(\mathrm{TN})$ is the Dirac bundle associated to the signature operator of Atiyah and Singer.

\section{SPIN ${ }^{\mathrm{c}}-$ STRUCTURES}

We shall define Spin $^{\mathrm{c}}$-structures using the notion of Dirac bundle that was introduced in the last section.

Definition 4.1. Denote by $\mathbb{C}_{n}$ the complex Clifford algebra for $\mathbb{R}^{n}$, generated by the standard basis elements $e_{1}, \ldots, e_{n}$ of $\mathbb{R}^{n}$.

Let $M$ be a smooth manifold and let $V$ be a rank $p$ Euclidean vector bundle over $M$. If $e_{1}, \ldots, e_{n}$ is a local orthonormal frame for $V$, defined over an open set $U \subseteq M$, then the trivial bundle $U \times \mathbb{C}_{n}$ over $U$ with fiber $\mathbb{C}_{n}$ may be given the structure of an $p$ graded Dirac bundle for $\left.\mathrm{V}\right|_{\mathrm{u}}$ : Clifford multiplication by an element $e_{j}$ of the frame is left multiplication by the $j$ th generator of $\mathbb{C}_{p}$, and the $p$-multigrading operators $\varepsilon_{1}, \ldots, \varepsilon_{p}$ for the bundle are right multiplication by the same generators.

Definition 4.2. Let $M$ be a smooth manifold and let $V$ be a $p$-dimensional Euclidean vector bundle over $M$. A complex spinor bundle for $V$ is a $p$-multigraded Dirac bundle $S_{V}$ which is locally isomorphic to the trivial bundle with fiber $\mathbb{C}_{p}$, the Clifford multiplication being determined from some local orthonormal frame, as above. We shall call a bundle $V$ equipped with a complex spinor bundle a $\operatorname{Spin}^{c}$-vector bundle. If $M$ is a smooth manifold (possibly with boundary) then by a $\operatorname{Spin}^{\mathrm{c}}$-structure on $M$ we shall mean a pair consisting of a Riemannian metric on $M$ and a complex spinor bundle $S_{M}$ for TM. 
Remark 4.3. A spinor bundle determines an orientation of $V$, as follows. If $\left\{f_{1}, \ldots, f_{p}\right\}$ is a local orthonormal frame for $V$, then the endomorphism of the spinor bundle $S_{V}$ determined by the formula

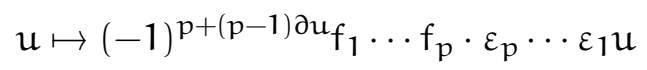

is plus or minus the identity (here $\partial u$ is the $\mathbb{Z} / 2$-grading degree of the section $u$ ). If the endomorphism is $+\mathrm{I}$ then we deem the frame to be oriented; if it is $-\mathrm{I}$ then we deem it to be oppositely oriented.

Example 4.4. Let $V_{1}$ and $V_{2}$ be Euclidean vector bundles on $M$ equipped with spinor bundles $S_{1}$ and $S_{2}$. Using the well-known Clifford algebra isomorphism $\mathbb{C}_{\mathrm{p}_{1}} \hat{\otimes} \mathbb{C}_{\mathrm{p}_{2}} \cong$ $\mathbb{C}_{p_{1}+p_{2}}$ the graded tensor product $S_{1} \hat{\otimes} S_{2}$ becomes a spinor bundle for $V_{1} \oplus V_{2}$. It defines the direct sum $\operatorname{Spin}^{\mathrm{c}}$-structure on $\mathrm{V}_{1} \oplus \mathrm{V}_{2}$.

Remark 4.5. The definition of $\mathrm{Spin}^{\mathrm{c}}$-structure can be rephrased in the language of principal bundles, as follows. The group $\operatorname{Spin}(n)$ is the closed subgroup of the unitary group of $\mathbb{C}_{n}$ whose Lie algebra is the $\mathbb{R}$-linear span of the elements $e_{i} e_{j}$, for $i \neq j$. The group $\operatorname{Spin}^{c}(n)$ is the closed subgroup of the unitary group of $\mathbb{C}_{n}$ which is generated by $\operatorname{Spin}(n)$ and the complex numbers of modulus one. The group $\operatorname{Spin}^{c}(n)$ acts by inner automorphisms on the $\mathbb{R}$-linear subspace of $\mathbb{C}_{n}$ spanned by the elements $e_{j}$, and in this way we obtain a homomorphism from $\operatorname{Spin}^{c}(n)$ into $G L(n, \mathbb{R})$ (in fact into $O(n)$ ). Now if $M$ is a smooth manifold, and if $P$ is a reduction to $\operatorname{Spin}^{c}(n)$ of the principal bundle of tangent frames, then the reduction determines a Riemannian metric on $M$, and the bundle

$$
\mathrm{S}=\mathrm{P} \times{ }_{\operatorname{Spin}^{\mathrm{c}}(\mathrm{n})} \mathbb{C}_{n}
$$

is a spinor bundle on $M$ (here $\operatorname{Spin}^{c}(n)$ acts on $\mathbb{C}_{n}$ by left multiplication). Thus $P$ determines a Spin ${ }^{c}$-structure. Conversely, every $\operatorname{Spin}^{c}$-structure arises in this way (up to isomorphism).

Definition 4.6. Let $M^{n}$ be a smooth manifold, without boundary, equipped with a Spin ${ }^{c}$ structure. We shall denote by $[M] \in K_{n}(M)$ the K-homology class of any Dirac operator on $\mathrm{S}$. This is the K-homology fundamental class of the $\operatorname{Spin}^{\mathrm{c}}$-manifold $\mathrm{M}$.

If $\bar{M}$ is a smooth manifold with boundary then of course a Riemannian metric on $\bar{M}$ restricts to one on the interior $M$, and also to one on the boundary $\partial M$. A spinor bundle $S$ for $\bar{M}$ restricts to a spinor bundle on $M$, and the boundary of $S$, as described in Definition 3.4, is a spinor bundle for $\partial M$. The following result is a consequence of Theorem 3.5.

Theorem 4.7. If $\mathrm{M}$ is the interior of an $\mathrm{n}$-dimensional $\mathrm{Spin}^{\mathrm{c}}$-manifold with boundary, and if we equip the boundary manifold $\partial \mathrm{M}$ with the induced $\mathrm{Spin}^{\mathrm{c}}$-structure, then the K-homology boundary map

$$
\partial: K_{n}(M) \rightarrow K_{n-1}(\partial M)
$$


takes the fundamental class of $\mathrm{M}$ to the fundamental class of $\partial \mathrm{M}$ :

$$
\partial[M]=[\partial M] \in K_{n-1}(\partial M) .
$$

Definition 4.8. Let $M$ be a smooth manifold equipped with a $\operatorname{Spin}^{\mathrm{c}}$-structure. The opposite Spin $^{\mathrm{c}}$-structure is defined by changing the action of the multigrading operator $\varepsilon_{1}$ by a sign.

Definition 4.9. Let $M$ be a smooth manifold. Two $\operatorname{Spin}^{c}$-structures on $M$ are concordant if there is a Spin ${ }^{c}$-structure on $[0,1] \times M$ for which the induced $\operatorname{Spin}^{c}$-structure on the boundary $M \cup M$ is one of the given $\operatorname{Spin}^{c}$ structures on one copy of $M$, and the opposite of the other given structure on the other copy of $M$.

In Chapter 11 of [HR00], the following result is proved.

Theorem 4.10. Concordant $\mathrm{Spin}^{\mathrm{c}}{ }^{\mathrm{s}}$-structures on $\mathrm{M}$ determine the same fundamental class in K-homology.

In the case of even-dimensional manifolds the following simplified description of Spin $^{\mathrm{c}}$-structures will be useful for us.

Definition 4.11. Let $M^{n}$ be a smooth, even-dimensional manifold. A reduced $\operatorname{Spin}^{\mathrm{c}}$ structure on $M$ consists of a Riemannian metric on $M$ and a Dirac bundle $S$ ( $\mathbb{Z} / 2$-graded, but with no $n$-grading structure) whose fiber dimension is $2^{\frac{n}{2}}$. We shall call $S$ a reduced spinor bundle.

If $n$ is even then the complex Clifford algebra $\mathbb{C}_{n}$ is isomorphic to the matrix algebra $M_{2^{\frac{n}{2}}}(\mathbb{C})$, and hence has a unique representation $V_{n}$ of dimension $2^{\frac{n}{2}}$. The operator

$$
\gamma=i^{\frac{n}{2}} e_{1} \cdots e_{n}
$$

provides $V_{n}$ with a $\mathbb{Z} / 2$-grading. If $S$ is a reduced spinor bundle, as in the definition, then the tensor product $S \hat{\otimes} V_{n}$ is a spinor bundle in the sense of Definition 4.2, and conversely every spinor bundle in the sense of Definition 4.2 is of this form. If we temporarily denote by $[M]_{\text {red }} \in K_{0}(M)$ the K-homology class of the Dirac operator on the reduced spinor bundle $S$, then under the periodicity map $K_{0}(M) \rightarrow K_{n}(M)$ the fundamental class $[M]_{\text {red }}$ maps to $[M]$.

We conclude this section by comparing reduced spinor bundles with the Dirac bundles Cliff $_{\frac{1}{2}}(T N)$ that we introduced in Section 3.

Let $\mathrm{N}$ be an even-dimensional, oriented Riemannian manifold, and assume it admits a Spin $^{\mathrm{c}}$-structure, with reduced spinor bundle $\mathrm{S}$. As we noted above, the complex Clifford algebra of a Euclidean vector space of dimension $n=2 k$ is isomorphic to the algebra 
of complex $2^{\mathrm{k}} \times 2^{\mathrm{k}}$ matrices. It follows by counting dimensions that the natural map $\operatorname{Cliff}(\mathrm{TN}) \rightarrow \operatorname{End}(\mathrm{S})$ is an isomorphism. Hence there is an isomorphism

$$
\operatorname{Cliff}(\mathrm{TN}) \cong \mathrm{S} \hat{\otimes} \mathrm{S}^{*}
$$

compatible with the left and right actions by Clifford multiplication.

Proposition 4.12. Let $\mathrm{S}$ be a reduced spinor bundle for $\mathrm{N}$ and denote by $\mathrm{S}_{+}^{*}$ the evengraded part of its dual. There is an isomorphism of Dirac bundles

$$
\mathrm{Cliff}_{\frac{1}{2}}(\mathrm{TN}) \cong \mathrm{S} \otimes \mathrm{S}_{+}^{*} .
$$

Proof. The reduced spinor bundle determines a full spinor bundle for $M$, which in turn determines the orientation of $M$, as described earlier. Having fixed this orientation, the operator $\gamma$ acts as +1 on $S_{+}$and -1 on $S_{-}$. So the proposition follows from the isomorphism $\operatorname{Cliff}(\mathrm{TN}) \cong \mathrm{S} \hat{\otimes} \mathrm{S}^{*}$.

\section{Review of GeOMETRIC K-Homology}

Definition 5.1. Let $X$ be a paracompact Hausdorff space and let $Y$ be a closed subspace of $\mathrm{X}$. A K-cycle for the pair $(X, Y)$ is a triple $(M, E, \phi)$ consisting of:

(i) A smooth, compact manifold $M$ (possibly with boundary), equipped with a Spin ${ }^{\mathrm{c}}$ structure.

(ii) A smooth, Hermitian vector bundle $E$ on $M$.

(iii) A continuous map $\phi: M \rightarrow X$ such that $\phi[\partial M] \subseteq Y$.

Remark 5.2. The manifold $M$ need not be connected. Moreover the components of $M$ may have differing dimensions.

Two K-cycles are isomorphic if there are compatible isomorphisms of all of the above three components in the definition of K-cycle (this includes an isomorphism of spinor bundles). Following [BD82] we are going to construct an abelian group from sets of isomorphism classes of cycles so as to obtain "geometric" K-homology groups for the pair $(X, Y)$. In order to define the relations in these groups we need to introduce several kinds of operations and relations involving K-cycles.

Definition 5.3. If $(M, E, \phi)$ and $\left(M^{\prime}, E^{\prime}, \phi^{\prime}\right)$ are two $K$-cycles for $(X, Y)$, then their disjoint union is the $\mathrm{K}$-cycle $\left(M \cup M^{\prime}, E \cup E^{\prime}, \phi \cup \phi^{\prime}\right)$.

Definition 5.4. If $(M, E, \phi)$ is a $\mathrm{K}$-cycle for $(X, Y)$, then its opposite is the K-cycle $(-M, E, \phi)$, where $-M$ denotes the manifold $M$ equipped with the opposite Spin $^{c}$ structure.

Definition 5.5. A bordism of $\mathrm{K}$-cycles for the pair $(\mathrm{X}, \mathrm{Y})$ consists of the following data:

(i) A smooth, compact manifold L, equipped with a $\mathrm{Spin}^{\mathrm{c}}$-structure.

(ii) A smooth, Hermitian vector bundle F over L. 
(iii) A continuous map $\Phi: L \rightarrow X$.

(iv) A smooth map $f: \partial L \rightarrow \mathbb{R}$ for which \pm 1 are regular values, and for which $\Phi\left[f^{-1}\right.$ $[-1,1]] \subseteq \mathrm{Y}$.

To understand the definition, it is best to consider the case where $Y=\emptyset$. In this case it follows from condition (iv) that the set $f[-1,1]$ is empty, and therefore the boundary of $L$ is divided by $f$ into two components: $M_{+}=f^{-1}(+1,+\infty)$ and $M_{-}=f^{-1}(-\infty,-1)$. We therefore obtain two K-cycles $\left(M_{+},\left.F\right|_{M_{+}},\left.\Phi\right|_{M_{+}}\right)$and $\left(M_{-},\left.F\right|_{M_{-}},\left.\Phi\right|_{M_{-}}\right)$, and we shall say that the first is bordant to the opposite of the second.

In the case where $Y$ is non-empty the sets $M_{+}=f^{-1}[+1,+\infty)$ and $M_{-}=f^{-1}$ $(-\infty,-1]$ are manifolds with boundary, and we obtain, as before two K-cycles $\left(M_{+}\right.$, $\left.\left.F\right|_{M_{+}},\left.\Phi\right|_{M_{+}}\right)$and $\left(M_{-},\left.F\right|_{M_{-}},\left.\Phi\right|_{M_{-}}\right)$, but now for the pair $(X, Y)$. Once again we shall say that the first is bordant to the opposite of the second.

The purpose of the function $f$ in Definition 5.5 is to provide a notion of bordism for manifolds with boundary without having to introduce manifolds with corners. Bordism is an equivalence relation.

We have one more operation on $\mathrm{K}$-cycles to introduce. Let $M$ be a Spin ${ }^{\mathrm{c}}$-manifold and let $W$ be a Spin ${ }^{c}$-vector bundle over $M$. Denote by $\mathbf{1}$ the trivial, rank-one real vector bundle. The direct sum $W \oplus \mathbf{1}$ is a Spin ${ }^{\mathrm{c}}$-vector bundle, and moreover the total space of this bundle may be equipped with a $\operatorname{Spin}^{c}$ structure in a canonical way, up to concordance. This is because its tangent bundle fits into an exact sequence

$$
0 \longrightarrow \pi^{*}[\mathrm{~W} \oplus \mathbf{1}] \longrightarrow \mathrm{T}(\mathrm{W} \oplus \mathbf{1}) \longrightarrow \pi^{*}[\mathrm{TM}] \longrightarrow 0,
$$

where $\pi$ is the projection from $W \oplus \mathbf{1}$ onto $M$, so that, upon choosing a splitting, (or equivalently, choosing a Riemannian metric on the manifold $W \oplus \mathbf{1}$ which is compatible with the above sequence) we have a direct sum decomposition

$$
\mathrm{T}(\mathrm{W} \oplus \mathbf{1}) \cong \pi^{*}[\mathrm{~W} \oplus \mathbf{1}] \oplus \pi^{*}[\mathrm{TM}] .
$$

Different splittings result in concordant $\operatorname{Spin}^{\mathrm{c}}$-structures.

Let us now denote by $Z$ the unit sphere bundle of the bundle $W \oplus \mathbf{1}$. Since $Z$ is the boundary of the disk bundle, we may equip it with a natural $\operatorname{Spin}^{\mathrm{c}}$-structure by first restricting the given $\operatorname{Spin}^{c}$-structure on total space of $W \oplus \mathbf{1}$ to the disk bundle, and then taking the boundary of this $\operatorname{Spin}^{\mathrm{c}}$-structure to obtain a $\operatorname{Spin}^{\mathrm{c}}$-structure on the sphere bundle.

Definition 5.6. Let $(M, E, \phi)$ be a K-cycle for $(X, Y)$ and let $W$ be a Spin ${ }^{c}$-vector bundle over $M$ with even-dimensional fibers. Let $Z$ be the sphere bundle of $W \oplus \mathbf{1}$, as above. The vertical tangent bundle of $Z$ has a natural $\operatorname{Spin}^{c}$-structure (one applies the boundary construction of Definition 3.4 to the pullback of $W \oplus \mathbf{1}$ to Z). Denote by $S_{V}$ the corresponding reduced spinor bundle and let $F=S_{V,+}^{*}$. In other words, define $F$ to be the dual of the even-graded part of the $\mathbb{Z} / 2$-graded bundle $S_{V}$. The modification of $(M, E, \phi)$ associated to $W$ is the $K$-cycle $\left(Z, F \otimes \pi^{*} E, \phi \circ \pi\right)$. 
We are now ready to define the Baum-Douglas geometric K-homology groups.

Definition 5.7. Denote by $K^{\text {geom }}(X, Y)$ the set of equivalence classes of $K$-cycles over $(X, Y)$, for the equivalence relation generated by the following relations:

(i) If $\left(M, E_{1}, \phi\right)$ and $\left(M, E_{2}, \phi\right)$ are two K-cycles with the same $\operatorname{Spin}^{c}$-manifold $M$ and map $\phi: M \rightarrow X$, then

$$
\left(M \cup M, E_{1} \cup E_{2}, \phi \cup \phi\right) \sim\left(M, E_{1} \oplus E_{2}, \phi\right) .
$$

(ii) If $\left(M_{1}, E_{1}, \phi_{1}\right)$ and $\left(M_{2}, E_{2}, \phi_{2}\right)$ are bordant $K$-cycles then

$$
\left(M_{1}, E_{1}, \phi_{1}\right) \sim\left(M_{2}, E_{2}, \phi_{2}\right) \text {. }
$$

(iii) If $(M, E, \phi)$ is a $\mathrm{K}$-cycle, and if $W$ is an even-dimensional Spin $^{\mathrm{c}}$-vector bundle over $M$, then

$$
(\mathrm{M}, \mathrm{E}, \phi) \sim\left(\mathrm{Z}, \mathrm{F} \otimes \pi^{*} \mathrm{E}, \phi \circ \pi\right),
$$

where $\left(Z, F \otimes \pi^{*} E, \phi \circ \pi\right)$ is the modification of $(M, E, \phi)$ given in Defintion 5.6.

The set $K^{\text {geom }}(X, Y)$ is in fact an abelian group. The addition operation is given by disjoint union,

$$
\left[M_{1}, E_{1}, \phi_{1}\right]+\left[M_{2}, E_{2}, \phi_{2}\right]=\left[M_{1} \cup M_{2}, E_{1} \cup E_{2}, \phi_{1} \cup \phi_{2}\right],
$$

and the additive inverse of a cycle is obtained by reversing the $\operatorname{Spin}^{\mathrm{c}}$-structure:

$$
-[\mathrm{M}, \mathrm{E}, \phi]=[-\mathrm{M}, \mathrm{E}, \phi] \text {. }
$$

The neutral element is represented by the empty manifold, or any cycle bordant to the empty manifold.

Definition 5.8. Denote by $K_{\mathrm{ev}}^{\text {geom }}(X, Y)$ and $K_{\text {odd }}^{\text {geom }}(X, Y)$ the subgroups of the group $K^{\text {geom }}(X, Y)$ composed of equivalence classes of $K$-cycles $(M, E, \phi)$ for which every connected component of $M$ is even dimensional and odd dimensional, respectively.

The groups $\mathrm{K}_{\mathrm{ev} / \mathrm{odd}}^{\mathrm{geom}}(\mathrm{X}, \mathrm{Y})$ are functorial in $(\mathrm{X}, \mathrm{Y})$, and they satisfy weak excision: if $\mathrm{U}$ is an open subset of $\mathrm{Y}$ whose closure is in the interior of $\mathrm{Y}$, then

$$
\mathrm{K}_{\mathrm{ev} / \mathrm{odd}}^{\mathrm{geom}}(\mathrm{X} \backslash \mathrm{U}, \mathrm{Y} \backslash \mathrm{U}) \cong \mathrm{K}_{\mathrm{ev} / \mathrm{odd}}^{\mathrm{geom}}(\mathrm{X}, \mathrm{Y}) \text {. }
$$

There is moreover a "homology sequence"

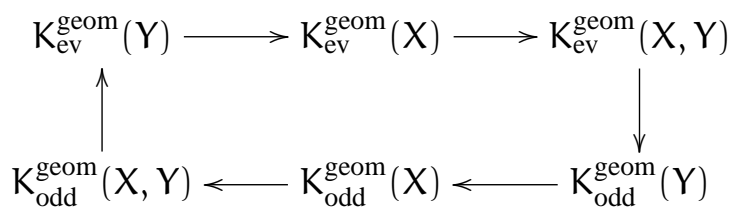

(where as usual we define $K_{\mathrm{ev}}^{\text {geom }}(Y)=K_{\mathrm{ev}}^{\text {geom }}(\mathrm{Y}, \emptyset)$, and so on). The boundary maps take a $\mathrm{K}$-cycle $(M, E, \phi)$ for $(X, Y)$ to the boundary cycle $\left(\partial M,\left.E\right|_{\partial M},\left.\phi\right|_{\partial M}\right)$ for $Y$ (it is easily verified that this definition is compatible with the equivalence relation used to define the geometric K-homology groups). The composition of any two successive 
arrows is zero. However it is not obvious that the sequence is exact. For the special case of finite CW-pairs this exactness will follow from the main theorem of the paper, which identifies geometric K-homology with Kasparov K-homology.

\section{Natural Transformation And Formulation of the Main TheOrem}

Now let $(X, Y)$ be a pair of compact and metrizable spaces. We associate to each K-cycle $(M, E, \phi)$ for $(X, Y)$ a class $\langle M, E, \phi\rangle$ in Kasparov K-homology, as follows. Denote by $M^{\circ}$ the interior of $M$, which is an open $\operatorname{Spin}^{\mathrm{c}}$-manifold. The Spin ${ }^{\mathrm{c}}$-structure on $M$ determines a spinor bundle $S$ on $M^{\circ}$ by restriction, and of course the complex vector bundle $E$ also restricts to $M^{\circ}$. The tensor product $S \otimes E$ is a Dirac bundle over $M^{\circ}$, and if $D_{E}$ is an associated Dirac operator, then we can form the class

$$
\left[D_{E}\right] \in K_{n}\left(M^{\circ}\right)
$$

(here $\mathrm{n}$ is the dimension of $M$ ). The map $\phi: M \rightarrow X$ restricts to a proper map from $M^{\circ}$ into $X \backslash Y$, and we can therefore form the class

$$
\phi_{*}\left[D_{E}\right] \in K_{n}(X, Y) .
$$

Theorem 6.1. The correspondence $(M, E, \phi) \mapsto \phi_{*}\left[D_{E}\right]$ determines a functorial map

$$
\mu: \mathrm{K}_{\mathrm{ev} / \text { odd }}^{\mathrm{geom}}(\mathrm{X}, \mathrm{Y}) \rightarrow \mathrm{K}_{\mathrm{ev} / \text { odd }}(X, Y)
$$

which is compatible with boundary maps in geometric and analytic K-homology.

Proof. The only thing to check is that the correspondence is compatible with the relations in Definition 5.7 which generate the equivalence relation on cycles used to define geometric K-homology. Once this is done, functoriality will be clear from the construction of $\langle M, E, \phi\rangle$ and compatibility with boundary maps will follow from Theorem 3.5.

Compatibility with relation (i) from Definition 5.7 is straightforward. Compatibility with relation (ii) follows from Theorem 3.5. So the proof reduces to showing that the correspondence is compatible with the relation (iii) of vector bundle modification.

Let $(M, E, \phi)$ be a $K$-cycle for $(X, Y)$ and let $n=\operatorname{dim}(M)$ (by working with one component of $M$ at a time we can assume that $\operatorname{dim}(M)$ is well-defined). Let $W$ be a $S_{\text {Spin }}{ }^{\mathrm{c}}$-vector bundle over $M$ of even fiber dimension $2 k$. Let $S_{M}$ be the spinor bundle for $M$, and let $S_{V}$ be the reduced spinor bundle for the vertical tangent bundle of the sphere bundle $\pi: Z \rightarrow M$. Form the tensor product

$$
S_{Z}=S_{V} \hat{\otimes} \pi^{*}\left[S_{M}\right] .
$$

This is neither a fully multigraded spinor bundle for $Z$ nor a reduced spinor bundle, but something in between. If $D_{Z}$ is a Dirac operator for $S_{Z}$ then the class $\left[D_{Z}\right] \in K_{n}(Z)$ is the image of the K-homology fundamental class $[Z] \in K_{n+2 k}(Z)$ under the periodicity isomorphism $K_{n+2 k}(Z) \cong K_{n}(Z)$. Similarly, if $D_{Z, F \otimes \pi * E}$ is a Dirac operator for the tensor product bundle $S_{Z} \otimes F \otimes \pi^{*} E$, then the class $\left[D_{Z, F \otimes \pi * E}\right] \in K_{n}(Z)$ is the image of 
the K-homology class of the modification $\left(Z, F \otimes \pi^{*} E, \phi Z\right)$ of the cycle $(M, E, \phi)$ under the same periodicity isomorphism.

To prove compatibility with the relation (iii) in the definition of geometric K-homology we need to show that $\left[D_{Z, F \otimes \pi_{*} E}\right]$ is equal to the class $\left[D_{M, E}\right] \in K_{n}(M)$. But writing

$$
S_{Z} \otimes F \otimes \pi^{*} E \cong\left[S_{V} \otimes F\right] \hat{\otimes} \pi^{*}\left[S_{M} \otimes E\right],
$$

we see that this follows from Propositions 3.6, 3.11 and 4.12.

We can now state the main theorem in this paper.

Theorem 6.2. If $(\mathrm{X}, \mathrm{Y})$ is a finite $\mathrm{CW}$-pair then the homomorphism

$$
\mu: \mathrm{K}_{\mathrm{ev} / \mathrm{odd}}^{\mathrm{geom}}(\mathrm{X}, \mathrm{Y}) \rightarrow \mathrm{K}_{\mathrm{ev} / \mathrm{odd}}(\mathrm{X}, \mathrm{Y})
$$

is an isomorphism.

The proof will be carried out in the remaining sections.

\section{Outline of THE PROOF}

We wish to prove that if $\mathrm{X}$ is a finite $\mathrm{CW}$ complex, then the homomorphisms

$$
\mu: \mathrm{K}_{\mathrm{ev} / \text { odd }}^{\mathrm{geom}}(\mathrm{X}) \rightarrow \mathrm{K}_{\mathrm{ev} / \text { odd }}(\mathrm{X})
$$

are isomorphisms. What makes this tricky is that we don't yet know that geometric Khomology is a homology theory. To get around this problem we are going to define a "technical" homology theory $k_{\mathrm{ev}}$ / odd $(X, Y)$ which fits into a commuting diagram

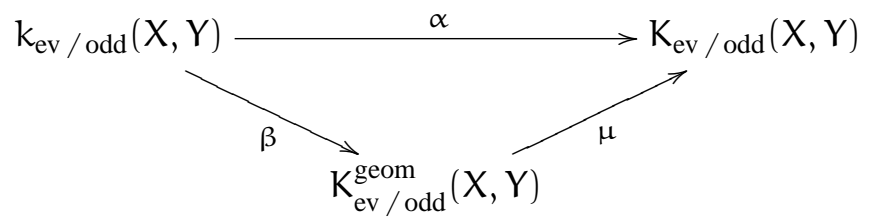

in which the horizontal arrow is a natural transformation between homology theories. Having done so, the proof will be completed in two steps:

(a) We shall check that when $X$ is a point and $Y$ is empty, the horizontal arrow is an isomophism. It will follow that the horizontal arrow is an isomorphism for every finite $\mathrm{CW}$ pair $(X, Y)$.

(b) We shall prove that for every finite $\mathrm{CW}$ pair $(X, Y)$, the map in the diagram from $k_{\mathrm{ev} / \text { odd }}(X, Y)$ to $K_{\mathrm{ev} / \text { odd }}^{\mathrm{geom}}(X, Y)$ is surjective.

It is clear that (a) and (b) together will imply that all the arrows in the diagram are isomorphisms, for every finite $\mathrm{CW}$ pair $(\mathrm{X}, \mathrm{Y})$.

The reader who is acquainted with the definition of K-homology starting from the Bott spectrum will see that our definition of $k_{\mathrm{ev}}$ / odd $(X, Y)$ is extremely close to the spectrum 
definition of K-homology. However the definition which is presented in the next section is not designed with this in mind.

\section{Definition of the Technical Group}

Fix a model $\mathbb{K}$ for the Oth space of the Bott spectrum, e.g. $\mathbb{Z} \times$ BU. We shall use the following features of this space:

(a) If $\mathrm{X}$ is a pointed finite $\mathrm{CW}$ complex, then there is a natural isomorphism

$$
\mathrm{K}^{\mathrm{O}}(\mathrm{X}, *) \cong[\mathrm{X}, \mathbb{K}]^{+}
$$

between the relative Atiyah-Hirzebruch $\mathrm{K}$-theory group $\mathrm{K}^{\mathrm{O}}(\mathrm{X}, *)$ and the set of homotopy classes of maps from $X$ into $\mathbb{K}$. Here $*$ is the base point of $X$ and $[X, \mathbb{K}]^{+}$ denotes the set of homotopy classes of basepoint-preserving maps (recall that $\mathbb{K}$ is a base-pointed space).

(b) There is a basepoint-preserving map $m: \mathbb{K} \wedge \mathbb{K} \rightarrow \mathbb{K}$ which induces the operation of tensor product (the ring structure) on $\mathrm{K}^{0}(\mathrm{X})$.

Example 8.1. We could take $\mathbb{K}$ to be the space of all Fredholm operators on a separable, infinite-dimensional Hilbert space $\mathrm{H}$ (the Fredholm operators are topologized by the operator-norm topology). The isomorphism (a) is described in [Ati67]. For later use we note that the set of connected components of $\mathbb{K}$ is isomorphic to $\mathbb{Z}$, the isomorphism being given by the Fredholm index.

Definition 8.2. Let $S^{2}$ be the standard 2-sphere, equipped with its standard $\operatorname{Spin}^{\mathrm{c}}$ structure as the boundary of the ball in $\mathbb{R}^{3}$. Denote by $\beta: S^{2} \rightarrow \mathbb{K}$ a basepoint-preserving map which, under the isomorphism $\left[\mathrm{S}^{2}, \mathbb{K}\right]^{+} \cong \mathrm{K}^{0}\left(\mathbb{R}^{2}\right)=\mathrm{K}^{0}\left(\mathrm{~S}^{2}, *\right)$, corresponds to the difference $\left[S_{+}^{*}\right]-[\mathbf{1}]$. Here $S_{+}^{*}$ is the dual of the positive part of the reduced spinor bundle on the 2 -sphere (which is the one-point compactification of $\mathbb{R}^{2}$ ), and $\mathbf{1}$ is the trivial line bundle.

Remark 8.3. Note that $S_{+}^{*}$ is a line bundle, so that the difference $\left[S_{+}^{*}\right]-[\mathbf{1}]$ has virtual dimension zero.

Now, we are going to construct the "technical" homology groups $k_{\mathrm{ev}}$ / odd $(X, Y)$ using the space $\mathbb{K}$, the map $\beta$, and the notion of framed bordism, which we briefly review.

Definition 8.4. A framed manifold is a smooth, compact manifold $M^{n}$ with a given stable trivialization ${ }^{4}$ of its tangent bundle:

$$
\mathbf{k} \oplus \mathrm{TM} \cong \mathbf{k} \oplus \mathbf{n}
$$

We shall identify two stable trivializations if they are stably homotopic (that is, homotopic after forming the direct sum with the identity map on an additional trivial summand). Thus a framed manifold is a smooth, compact manifold together with a stable homotopy class of stable trivializations of its tangent bundle.

\footnotetext{
${ }^{4}$ In this definition we are using $\mathbf{n}$ or $\mathbf{k}$ to denote the trivial real vector bundle of rank $n$ or $k$, respectively.
} 
Definition 8.5. If $(X, Y)$ is any paracompact and Hausdorff pair then we shall denote by $\Omega_{n}^{F}(X, Y)$ the $n$-th framed bordism group of the pair $(X, Y)$. Thus $\Omega_{n}^{F}(X, Y)$ is the set of all bordism classes of maps from framed manifolds into $X$, mapping the manifold boundaries into Y. Compare Definition 5.5 or [Sto68]. Note that the boundary of a framed manifold $M$ is itself a framed manifold in a natural way: starting from a stable trivialization

$$
\mathbf{k} \oplus \mathrm{TM} \cong \mathbf{k} \oplus \mathbf{n}
$$

we use an inward pointing normal vector field on $\partial M$ to obtain a stable trivialization

$$
\mathbf{k} \oplus \mathbf{1} \oplus \mathrm{T} \partial \mathrm{M} \cong \mathbf{k} \oplus \mathbf{n} .
$$

We use the inward pointing normal to agree with orientation conventions established earlier.

We can now define our "technical" homology theory $k_{\mathrm{ev}}$ /odd $(X, Y)$. For a finite CW pair $(X, Y)$ and an integer $n$, form a direct system of abelian groups

$$
\Omega_{\mathrm{n}}^{\mathrm{F}}(\mathrm{X} \times \mathbb{K}, \mathrm{Y} \times \mathbb{K}) \rightarrow \Omega_{\mathrm{n}+2}^{\mathrm{F}}(\mathrm{X} \times \mathbb{K}, \mathrm{Y} \times \mathbb{K}) \rightarrow \Omega_{\mathrm{n}+4}^{\mathrm{F}}(\mathrm{X} \times \mathbb{K}, \mathrm{Y} \times \mathbb{K}) \rightarrow \cdots
$$

as follows. Given a cycle $f: M \rightarrow X \times \mathbb{K}$ for $\Omega_{n+2 k}^{F}(X \times \mathbb{K}, Y \times \mathbb{K})$, the composition

$$
M \times S^{2} \stackrel{f \times \beta}{\longrightarrow} X \times \mathbb{K} \times \mathbb{K} \stackrel{1 \times m}{\longrightarrow} X \times \mathbb{K}
$$

is a cycle for $\Omega_{n+2 k+2}^{F}(X \times \mathbb{K}, Y \times \mathbb{K})$. This defines the map from $\Omega_{n+2 k}^{F}(X \times \mathbb{K}, Y \times \mathbb{K})$ to $\Omega_{n+2 k+2}^{\mathrm{F}}(\mathrm{X} \times \mathbb{K}, \mathrm{Y} \times \mathbb{K})$ which appears in the directed system.

Definition 8.6. Denote by $k_{\mathrm{ev} / \mathrm{odd}}(\mathrm{X}, \mathrm{Y})$ the direct limit of the above directed system, for $n$ even/odd.

Since $\Omega_{*}^{\mathrm{F}}$ is itself a homology theory (on finite CW pairs), and since direct limits preserve exact sequences, it is clear that $k_{*}$ is a homology theory.

The map $\beta$ from $k_{\mathrm{ev} / \mathrm{odd}}(\mathrm{X}, \mathrm{Y})$ into $\mathrm{K}_{\mathrm{ev} / \mathrm{odd}}^{\mathrm{geom}}(\mathrm{X}, \mathrm{Y})$ which appears in Section 7 is defined as follows (for notational simplicity we will only describe the construction for the absolute groups $k_{\mathrm{ev}}$ / odd $(X)$, not the relative groups). If $M$ is a framed manifold then the framing $\mathbf{k} \oplus \mathrm{TM} \cong \mathbf{k} \oplus \mathbf{n}$ determines a Spin ${ }^{\mathrm{c}}$-structure on $M$. A map $M \rightarrow X \times \mathbb{K}$ determines a map $\phi: M \rightarrow X$ and a K-theory class for $M$, which we may represent as a difference $\left[E_{1}\right]-\left[E_{2}\right]$ for some vector bundles $E_{1}$ and $E_{2}$. A map

$$
\beta_{n}: \Omega_{n}^{\mathrm{F}}(X \times \mathbb{K}) \rightarrow K_{n}^{\text {geom }}(X)
$$

is defined by associating to the bordism class of $M \rightarrow X \times \mathbb{K}$ the difference of K-cycles $\left(M, E_{1}, \phi\right)-\left(M, E_{2}, \phi\right)$. It follows from part (i) of Definition 5.7 that the K-homology class of this difference does not depend on the choice of $E_{1}$ and $E_{2}$ to represent the Ktheory class on $\mathrm{M}$. It follows from part (ii) of the definition that the K-homology class 
only depends on the bordism class of the map $M \rightarrow X \times \mathbb{K}$. Finally, it follows from part (iii) of the definition that the diagram

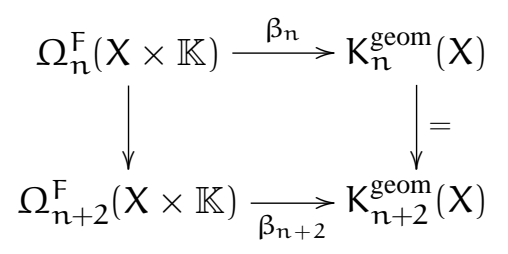

is commutative (on the right is the periodicity isomorphism described in Section 2). Since $\beta$ is compatible with the direct limit procedure using which $k_{\mathrm{ev}}$ /odd $(X)$ is obtained from the framed bordism groups, we obtain maps

$$
\beta: k_{\text {ev } / \text { odd }}(X) \rightarrow K_{\text {ev } / \text { odd }}^{\text {geom }}(X)
$$

as required.

\section{Proof of the Main Theorem}

9.1. Proof of (a). We wish to show that the maps $\alpha: k_{n}(p t) \rightarrow K_{n}(p t)$ are isomorphisms for $n=0$ and $n=1$. If $W$ is any (base-pointed) topological space then by the Pontrjagin-Thom isomorphism [Pon42, Pon59, Tho54] the nth framed bordism group of $W$ is isomorphic to the nth stable homotopy group of $W: \Omega_{\mathfrak{n}}^{F}(W) \cong \pi_{\mathfrak{n}}^{S}(W)$. According to Bott periodicity, [Bot57, ?] the second loop space of $\mathbb{K}$ has the homotopy type of $\mathbb{K}$. In fact the map

$$
S^{2} \wedge \mathbb{K} \stackrel{\beta \wedge 1}{\longrightarrow} \mathbb{K} \wedge \mathbb{K} \stackrel{m}{\longrightarrow} \mathbb{K}
$$

induces a homotopy equivalence $\mathbb{K} \sim \Omega^{2} \mathbb{K}$. This, and the fact that the flip map $S^{2} \wedge$ $S^{2} \rightarrow S^{2} \wedge S^{2}$ is homotopic to the identity map, imply that the evident maps

$$
\lim _{\longrightarrow} \pi_{n+2 k}(\mathbb{K}) \rightarrow \lim _{\longrightarrow} \pi_{n+2 k}^{S}(\mathbb{K})
$$

are isomorphisms. The first direct limit is formed by associating to a map $f: S^{n+2 k} \rightarrow \mathbb{K}$ the composition

$$
S^{2} \wedge S^{n+2 k} \stackrel{1 \wedge f}{\longrightarrow} S^{2} \wedge \mathbb{K} \stackrel{m}{\longrightarrow} \mathbb{K}
$$

and the second direct limit is formed using a similar procedure, starting with maps from $S^{n+2 k+2 j}$ into $S^{2 j} \wedge \mathbb{K}$. To verify the assertion, view the second direct limit as the limit 
of the array

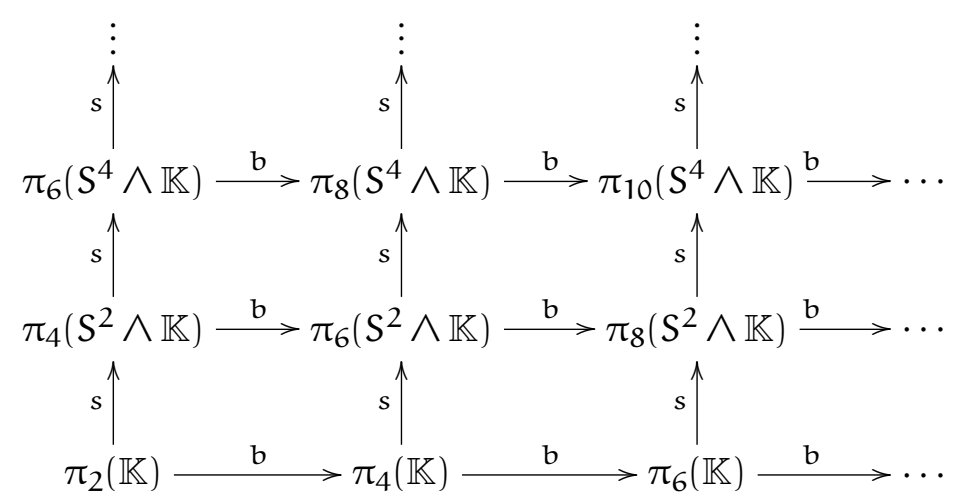

in which the vertical maps are suspension by $S^{2}$ and the horizontal maps are induced from suspension by $S^{2}$, followed by composition with $b: S^{2} \wedge \mathbb{K} \rightarrow \mathbb{K}$ defined by

$$
S^{2} \wedge \mathbb{K} \stackrel{\beta \wedge 1}{\longrightarrow} \mathbb{K} \wedge \mathbb{K} \stackrel{m}{\longrightarrow} \mathbb{K}
$$

The first direct limit is then the direct limit of the bottom row, and the required isomorphism follows from these facts:

(i) If $x \in \pi_{2 k}\left(S^{2 j} \wedge \mathbb{K}\right)$, and if $s(x)=0$, then $b(x)=0$.

(ii) If $x \in \pi_{2 k}\left(S^{2 j} \wedge \mathbb{K}\right)$ for some $j>0$, and if $x=b(y)$, for some $y \in \pi_{2 k-2}\left(S^{2 j} \wedge \mathbb{K}\right)$, then $x=s(z)$, for some $z \in \pi_{2 k-2}\left(S^{2 j-2} \wedge \mathbb{K}\right)$.

Item (i) is an immediate consequence of the definition of the map b. As for item (ii), if $x=b(y)$, then $x$ can be written as a composition

$$
S^{2} \wedge S^{2 k-2} \stackrel{1 \wedge y}{\longrightarrow} S^{2} \wedge S^{2 j} \wedge \mathbb{K} \stackrel{1 \wedge b}{\longrightarrow} S^{2 j} \wedge \mathbb{K}
$$

Writing $S^{2 j}$ as $S^{2 j-2} \wedge S^{2}$, and using the fact that the flip on $S^{2} \wedge S^{2}$ is homotopic to the identity, we can write this composition as

$$
S^{2} \wedge S^{2 k-2} \stackrel{1 \wedge y}{\longrightarrow} S^{2} \wedge S^{2 j-2} \wedge S^{2} \wedge \mathbb{K} \stackrel{1 \wedge 1 \wedge b}{\longrightarrow} S^{2} \wedge S^{2 j-2} \wedge \mathbb{K}
$$

This is clearly in the image of the map s.

Now

$$
k_{n}(p t)=\lim _{\longrightarrow} \Omega_{n+2 k}^{F}(\mathbb{K}) \cong \lim _{\longrightarrow} \pi_{n+2 k}^{S}(\mathbb{K}) \cong \lim _{\longrightarrow} \pi_{n+2 k}(\mathbb{K}) \cong \pi_{n}(\mathbb{K}) .
$$

As a result we obtain the isomorphisms

$$
\mathrm{k}_{\mathrm{n}}(\mathrm{pt}) \cong \pi_{\mathrm{n}}(\mathbb{K}) \cong \mathrm{K}^{0}\left(\mathbb{R}^{\mathrm{n}}\right)
$$

which implies that $k_{\text {ev }}(\mathrm{pt}) \cong \mathbb{Z}$ and $k_{\text {odd }}(\mathrm{pt})=0$. It follows immediately that the map $\alpha: \mathrm{k}_{\text {odd }}(\mathrm{pt}) \rightarrow \mathrm{K}_{\mathrm{odd}}(\mathrm{pt})$ is an isomorphism, since both domain and range are zero. In the 
even case the map

$$
\mathrm{k}_{0}(\mathrm{pt}) \cong \pi_{0}(\mathbb{K}) \rightarrow \mathrm{K}_{0}(\mathrm{pt}) \cong \mathbb{Z}
$$

sends a Fredholm operator $\mathrm{T}$ to the index of $\mathrm{T}$. This map is an isomorphism.

9.2. Proof of (b). We wish to prove that the map $k_{\mathrm{ev} / \mathrm{odd}}(\mathrm{X}, \mathrm{Y}) \rightarrow \mathrm{K}_{\mathrm{ev} / \mathrm{odd}}^{\mathrm{geom}}(\mathrm{X}, \mathrm{Y})$ is surjective. The image of this map consists precisely of the equivalence classes of $\mathrm{K}$ cycles $(N, F, \psi)$ for which $N$ is a framed $\operatorname{Spin}^{c}$-manifold. So we must prove that if $(M, E, \phi)$ is any $K$-cycle for $(X, Y)$, then there is an equivalent $K$-cycle $(N, F, \psi)$ for which $\mathrm{N}$ is a framed $\mathrm{Spin}^{\mathrm{c}}$-manifold.

To do this, choose a smooth real vector bundle $V$, with even-dimensional fibers, such that $\mathrm{TM} \oplus \mathrm{V}$ is trivializable, and fix an isomorphism

$$
\mathrm{TM} \oplus \mathrm{V} \cong \mathbf{n} \oplus \mathbf{k} .
$$

The trivial bundle $\mathbf{n} \oplus \mathbf{k}$ has a canonical Spin ${ }^{\mathrm{c}}$-structure, and the above isomorphism and the following lemma therefore define a Spin ${ }^{\mathrm{c}}$-structure on $\mathrm{V}$.

Lemma 9.1. Let $\mathrm{V}$ and $\mathrm{W}$ be real, orthogonal vector bundles over the same space $\mathrm{X}$. Assume that $\mathrm{V}$ and $\mathrm{V} \oplus \mathrm{W}$ are equipped with $\mathrm{Spin}^{\mathrm{c}}$-structures. There is a $\mathrm{Spin}^{\mathrm{c}}$-structure on $\mathrm{W}$ whose direct sum with the given $\mathrm{Spin}^{\mathrm{c}}$-structure on $\mathrm{V}$ is the given $\mathrm{Spin}^{\mathrm{c}}$-structure on $\mathrm{V} \oplus \mathrm{W}$.

Proof. Let $S_{V}$ be a (non-reduced) spinor bundle for $V$ and let $S_{V \oplus W}$ be the same for $V \oplus W$. Denote by $S_{W}$ the bundle of fiberwise linear maps $S_{V} \rightarrow S_{V \oplus W}$ which gradedcommute with the Clifford action of $V$ and which graded-commute with the action of the first $k$ multigrading operators $\varepsilon_{1}, \ldots, \varepsilon_{k}$, where $k=\operatorname{rank}(V)$. The bundle $W$ acts on $S_{V \oplus W}$, as do the remaining multigrading operators. By composition, $W$ and the remaining multigrading operators also act on $S_{W}$. A local consideration shows that we obtain a (non-reduced) spinor bundle for $\mathrm{W}$, and that it has the required property with respect to direct sum.

The vector bundle modification of the K-cycle $(M, E, \phi)$ by $V$ is a K-cycle whose manifold is framed, as required.

\section{Appendix: The Real Case}

In this appendix we briefly discuss the changes needed to prove the result analogous to Theorem 6.2 in KO-homology.

Kasparov's theory readily adapts to the real case. A real Hilbert space can be viewed as a complex Hilbert space equipped with a conjugate-linear isometric involution. A real $\mathrm{C}^{*}$-algebra is the same thing as a complex $\mathrm{C}^{*}$-algebra equipped with a conjugate linear involutive $*$-automorphism (which, unlike the $*$-operation, preserves the order of 
products). By including these complex-conjugation operators, the definitions of Section 2 extend immediately to the real case. The only difference is that in the real case the counterpart of the formal periodicity map $\mathrm{K}^{-\mathrm{p}} \rightarrow \mathrm{K}^{-(\mathrm{p}+2)}$ does not exist. However the four-fold composition of this map is compatible with real structures, and defines a real formal periodicity map $\mathrm{KO}^{-\mathrm{p}} \rightarrow \mathrm{KO}^{-(\mathrm{p}+8)}$. The results of Section 3 carry over without change, except that the bundle $\mathrm{Cliff}_{\frac{1}{2}}(\mathrm{TN})$ is a real Dirac bundle only when the dimension of $\mathrm{N}$ is a multiple of 4 . Our discussion of $\operatorname{Spin}^{\mathrm{c}}$ structures in Section 4 is designed to carry over to the real case just by replacing complex Clifford algebras with real Clifford algebras; reduced real spinor bundles exist in dimensions which are multiples of 8. The geometric definition of K-homology is based on Spin-manifolds-the real counterparts of $\mathrm{Spin}^{\mathrm{c}}$-manifolds-and the real counterpart of Theorem 6.2 is now easy to formulate. The only really new aspect of the proof is that a more careful treatment of part (a) is required. The argument given above shows that

$$
\mathrm{ko}_{0}(\mathrm{pt}) \cong \pi_{0}(\mathbb{K O}) \cong \mathrm{KO}^{0}\left(\mathbb{R}^{\mathrm{n}}\right) .
$$

Under these isomorphisms, the map $\mathrm{ko}_{0}(\mathrm{pt}) \rightarrow \mathrm{KO}^{\mathrm{n}}(\mathrm{pt})$ corresponds to the map $\mathrm{KO}^{0}$ $\left(\mathbb{R}^{\mathfrak{n}}\right) \rightarrow \mathrm{KO}_{\mathfrak{n}}(\mathrm{pt})$ which takes a K-theory class $\mathrm{x}$ to the index of the Dirac operator on $\mathbb{R}^{n}$ twisted by $x$. The fact that this map is an isomorphism is another formulation of Bott Periodicity (compare [Ati68]).

\section{REFERENCES}

[AB64] M.Atiyah and R.Bott. On the periodicity theorem for complex vector bundles. Acta Math., 112:229-247, 1964.

[Ati67] M.F.Atiyah. K-Theory.Benjamin Press. New Yok, 1967.

[Ati68] M.F.Atiyah.Bott periodicity and the index of elliptic operators. Quart.J.Math.Oxford Ser.(2), 19:113-140,1968.

[Ati70] M.F.Atiyah.Global theory of elliptic operators, In proc.of the International Symposium on Functional Analysis, Tokyo, 1970, Unversity of Tokyo Press.

[BCH94] P.Baum, A, Connes, and N.Higson, Classifying space for proper actions and K-theory of group C*-algebras, Contemporary mathematics, 167:241-291, 1994.

[BD82] P.Baum and R.Douglas, K-homology and index theory. In R Kadison,editor, Operator Algebras and Applications, volume 38 of proceedings of Symposia in Pure Mathematics, pages 117-173, Providence, RI, 1982. American Mathematical Society.

[BDF77] L.Brown, R.Douglas, and P.Fillmore. Extensons of $\mathrm{C}^{*}$-algebras and K-homology, Annals of Math., 105:265-324, 1977.

[BFM75] P.Baum, W.Fulton, and R.Macpherson. Riemann-Roch for singular varicties. Inst. Hautes Études Sci, Publ, Math.,(45):101-145, 1975. P.Baum, W.Fulton, and

[BFM79] R.Macpherson. Riemann-Roch and topological K theory for singular varieties. Acta Math., 143(3-4):155-192, 1979.

[Bot57] R.Bott.The stable homotopy of the classical groups, Proc.Nat.Acad.Sci, U.S.A.,43:933-935, 1957.

[HR00] N.Higson and J.Roe, Analytic K-homology, Oxford Mathematical Monographs. Oxford University Press, Oxford, 2000, Oxford Science Publications.

[Jak98] M.Jakob. A bordism-type description of homology. Manuscripta Math., 96(1):67-80, 1998.

[Jak00] M.Jakob. An alternative approach to homology, In Une dégustatum topologique [Topological morsels]:homotopy theory in the Swiss Alps(Arolla, 1999), volume 265 of Contemp.Math., pages 87-97. Amer Math.Soc., Providence, RI, 2000. 
[Kas75] G.G.Kasparov. Topological invariants of elliptic operators I: K-homology. Math.USSR Izvestija, 9:751-792, 1975.

[Pon42] L.Pontrjagin. Mappings of the three-dimensional sphere into an n-dimensional complex. C.R.(Doklady)Acad Sci.URSS(N.S), 34:35-37, 1942.

[Pon59] L.S.Pontryagin. Smooth manifolds and their applications in homotopy theory. In American Mathematical Society Translations, Ser 2, Vol.11, pages 1-114. American Mathematical Society, Providence. R.L., 1959.

[Sto68] R.E:Stong. Noes on cobordism theory. Mathematical notes. Princeton Unibersity Press, Princeton, N.J., 1968.

[Tho54] R.Thom. Quelques propriétés globales des variétés différentiables. Comment Math.Helv.,28:1786, 1954.

\section{Paul Baum}

Department of Mathematics

Penn State University

University Park, PA 16802

E-mail: baum@math.psu.edu

Nigel Higson

Department of Mathematics

Penn State University

University Park, PA 16802

E-mail: higson@math.psu.edu

Thomas Schick

Mathematisches Institut

Georg-August-Universität Göttingen

Bunsenstr. 3, D-37073 Göttingen, Germany

E-mail: schick@uni-math.gwdg.de 